Ann. Biol. anim. Bioch. Biophys., 1973, 13 (4), 691-697.

NOTE

\title{
PRÉPARATION D'ACIDE OLÉIQUE A 99,5 P. 100 DE PURETÉ
}

\author{
Lucie FRÉMONT et Marie-Thérèse GOZZELINO \\ Station de Recherches de Nutrition, \\ Centre national de Recherches zootechniques, I. N. R. A., \\ 78350 Jouy en Josas
}

\begin{abstract}
RÉSUMÉ
L'acide oléique est isolé à partir des esters méthyliques des acides gras de l'huile d'olive. L'utilisation de la technique des inclusions avec l'urée permet l'élimination des acides gras saturés et de la plus grande partie des acides gras insaturés. Après quatre cristallisations effectuées $\grave{a ̀ ~}+4^{\circ} \mathrm{C}$ ou à $+20^{\circ} \mathrm{C}$, il reste avec l'oléate de méthyle moins de $5 \mathrm{p}$. 100 d'autres esters méthyliques. Une dernière cristallisation des esters est effectuée dans l'acétone à $-60^{\circ} \mathrm{C}$. Enfin une hydrolyse des esters permet d'obtenir de l'acide oléique dont le degré de pureté est égal ou supérieur à 99,5 p. Ioo.
\end{abstract}

Pour connaitre les processus de formation et de métabolisation des glycérides dans les organes et les liquides circulants des animaux, il est nécessaire d'effectuer des études fines avec des produits de très grande pureté. Pour procéder à des expériences sur animaux, il est indispensable d'avoir à sa disposition de grandes quantités d'acides gras et de glycérides dont la composition et la structure soient parfaitement définies. Parmi les acides gras couramment trouvés dans le commerce, certains, comme l'acide palmitique, ont un degré de pureté suffisamment élevé pour que les acides gras «contaminants » puissent être éliminés facilement. L'acide oléique, en revanche, est très impur; à l'analyse apparaissent en effet non seulement de nombreux autres acides gras, mais aussi un taux élevé de produits de dégradation. Pour cette raison, nous avons été obligés de mettre au point, avec les moyens du laboratoire, une technique de préparation d'acide oléique pratiquement pur.

\section{IN'TRODUCTION}

Différentes techniques mettant en jeu les propriétés physiques des acides gras ou de leurs dérivés permettent d'isoler l'un d'eux à partir des triglycérides mixtes naturels.

L'une des plus utilisées est la distillation fractionnée basée sur la relation qui existe entre la longueur de chaîne des acides gras et leur point d'ébullition. Cependant, le degré de saturation 
ayant peu d'influence sur le point d'ébullition, il n'est pas possible de séparer complètement les acides gras saturés des acides gras insaturés de même longueur de chaîne. L'application de cette technique en laboratoire a fait l'objet de plusieurs publications parmi lesquelles nous citerons celles de MurRay (1955) et de Loury (I968).

La distillation est effectuée de préférence sur les esters méthyliques, car ceux-ci n'ont pas, comme les acides gras, tendance à former des dimères et leur point d'ébullition est inférieur à celui des acides gras correspondants. En opérant à pression réduite, les risques de décomposition thermique sont diminués, mais sous une pression de I $\mathrm{mmHg}$, il faut cependant chauffer de $100^{\circ} \mathrm{C}$ à $200^{\circ} \mathrm{C}$ pour séparer les esters de $\mathrm{C}_{14}$ à $\mathrm{C}_{20}$.

La distillation fractionnée est un procédé intéressant, mais comme elle ne permet qn'une séparation partielle des acides gras, elle est associée généralement à une autre technique. La méthode de fractionnement des acides gras par cristallisation dans les solvants organiques a été largement développée par Brown (Brown, I955; Brown et KoLB, 1955). Elle met en jeu les différences de solubilité à une température donnée des acides gras ou de leurs dérivés dans un solvant déterminé. La solubilité d'un acide gras dépend de la nature du solvant, de sa polarité et, pour un solvant donné, elle est en relation avec le point de fusion de l'acide. En général, les acides gras saturés à nombre pair d'atomes de carbone sont d'autant moins solubles que leur point de fusion est plus élevé ; pour les acides insaturés, la solubilité augmente avec le nombre de doubles liaisons. La solubilité est également affectée par la position des doubles liaisons et la configuration cis ou trans de l'acide. Loury (1968) obtient de l'acide oléique très pur en effectuant une distillation puis deux cristallisations dans l'acétone à $-35^{\circ} \mathrm{C}$ et à $-55^{\circ} \mathrm{C}$.

Pour séparer les acides gras selon leur degré de saturation, une technique basée sur les différences de solubilité des sels de plomb ou de lithium ou des savons " acides " de sodium peut. être aussi utilisée mais la séparation est imparfaite.

En 1940, BEngen a observé que l'urée pouvait former des "complexes " avec les chaînes aliphatiques normales. Par la suite, le principe et les propriétés de ces composés ont été étudiés en particulier par Schlenk, qui a montré que leur formation est en relation avec la forme moléculaire de la chaîne. Les techniques de fractionnement des acides gras décrites par les auteurs (Schlenk et Holman, I950 ; Schlenk, I954; Schlenk, I961 ; SWern et Parker, 1952 ; Swern, 1964) sont maintenant largement utilisées.

Le principe de ces inclusions est fondé sur la forme de cristallisation de l'urée et de ses dérivés. L'urée seule cristallise en prismes tétragonaux. En présence de chaînes aliphatiques normales, les molécules d'urée forment des prismes à base hexagonale ; les angles sont occupés par les molécules d'urée et la lumière reçoit la molécule " hôte ". Pour que l'inclusion soit stable, il faut que la chaîne de l'" hôte " ait au moins 6 atomes de carbone et qu'elle ne soit pas ramifiée. L'ensemble étant facilement décomposé par un acide dilué, il est possible de récupérer la chaîne incluse. Par cette technique, il est aisé de séparer les acides gras à courte chaine des acides gras à longue chaîne et, parmi ces derniers, les acides gras à chaîne linéaire des acides gras à chaîne ramifiée. Les acides gras saturés et les acides gras insaturés peuvent être également séparés car la facilité à former des inclusions avec l'urée est plus grande pour les premiers. Les avantages de cette technique sont multiples; les acides gras n'ont pas à être chauffés et l'inclusion les protège de l'oxydation ; de plus, la technique est peu coûteuse, donne un meilleur rendement que la distillation fractionnée et est facile à mettre en œuvre.

L'huile d'olive qui contient au moins $70 \mathrm{p}$. Ioo d'acide oléique est la matière première de choix. Après une série de cristallisations à l'urée, les acides gras insaturés accompagnant l'acide oléique sont éliminés par un procédé physique ou chimique : distillation ou cristallisation fractionnée (Schlenk et Holman, 1950; Swern et Parker, 1952 ; Fedeli, Camurati et Lanzani, I969), traitement par l'anhydride maléique et l'iode (KEPPLER et al., 1959), cristallisation des savons " acides" (Rubin et PAIsLey, 1960).

Dans la méthode que nous utilisons, le principe des inclusions avec l'urée est appliqué aux esters méthyliques des acides gras de l'huile d'olive; par modification des techniques proposées par les auteurs précités, nous obtenons un acide oléique dont la pureté est de l'ordre de 99,5 p. roo. 


\section{PARTIE EXPÉRIMENTALE}

\section{Principe}

Au cours des cristallisations successives, la majeure partie de l'acide oléique se trouve soit dans les cristaux, soit dans le filtrat selon la température de cristallisation et la dilution (fig. I).

Une première cristallisation à $4^{\circ} \mathrm{C}$ élimine la majorité des acides saturés. L'addition d'urée au filtrat provoque après dissolution et refroidissement à $4^{\circ} \mathrm{C}$ la cristallisation d'une partie des

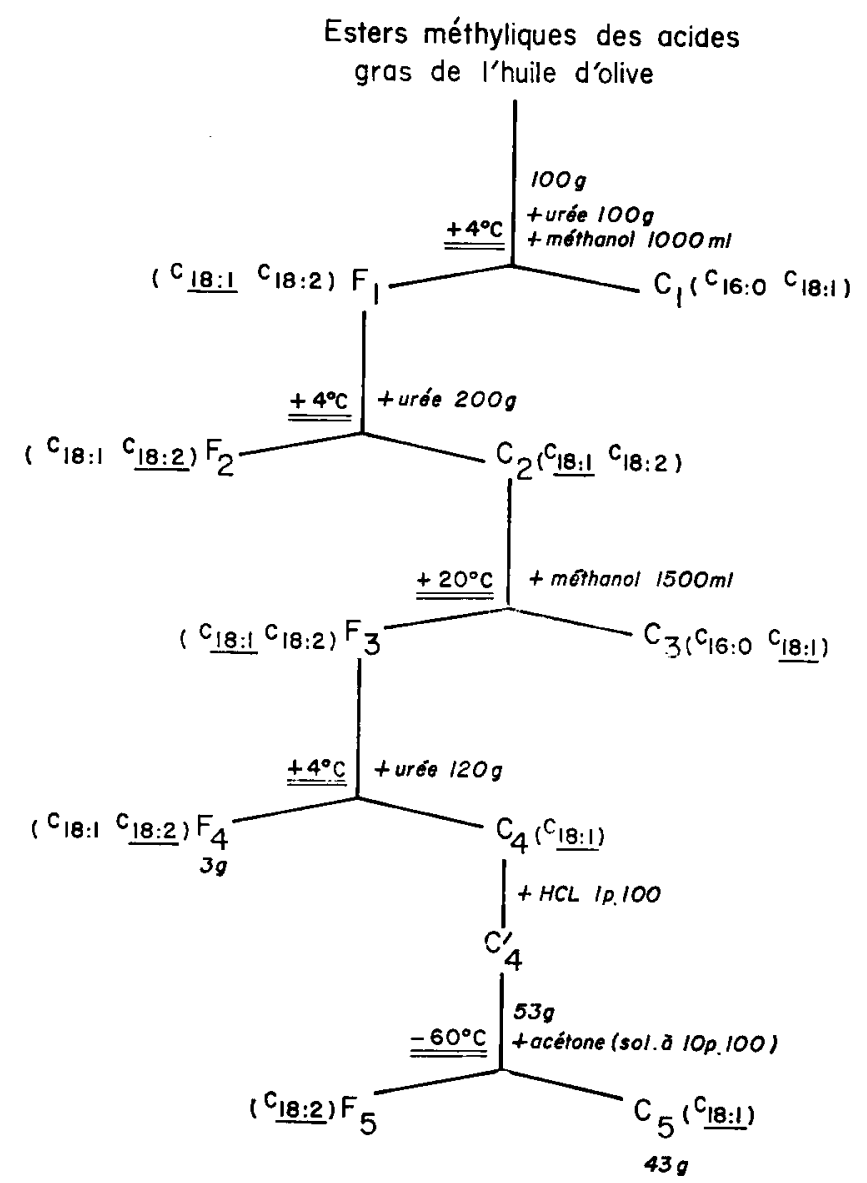

FIG. I. - Préparation de l'oléate de méthyle par cristallisation fractionnée des esters méthyliques de l'huile d'olive

Les acides gras se trouvant en majorité dans les différentes fractions sont indiqués entre les parenthèses.

L'acide gras souligné est le constituant principal.

acides saturés restants, de l'acide oléique et d'une fraction des autres acides insaturés. Afin d'éliminer de ces cristaux les acides saturés, on provoque la formation d'inclusions à partir d'une solution très diluée et à température plus élevée. De cette façon, il se forme peu d'inclusions ; les acides saturés qui sont en faible quantité cristallisent ,alors que l'acide oléique reste en majorité dans le filtrat, bien que sa proportion dans les inclusions soit élevée.

L'addition d'urée au filtrat et le refroidissement à $4^{\circ} \mathrm{C}$ provoque la cristallisation de l'acide oléique. Les inclusions contiennent encore de petites quantités d'acide palmitique et d'acides 
insaturés. Pour éliminer ces derniers, l'urée est séparée des esters méthyliques et ceux-ci, mis en solution dans l'acétone, sont refroidis à $-60^{\circ} \mathrm{C}$. Ainsi, l'oléate de méthyle cristallise avec une quantité d'acide palmitique inférieure à o,5 p. Ioo.

\section{Mode opératoive}

Préparation des esters méthyliques.

La transméthylation des triglycérides de l'huile d'olive est effectuée par le méthylate de sodium. Ce composé est préparé extemporanément en ajoutant environ I g de sodium à roo $\mathrm{ml}$ de méthanol anhydre. (La présence d'eau provoquerait une hydrolyse et la formation de savons.) Sur un ballon contenant $200 \mathrm{ml}$ d'huile d'olive, $100 \mathrm{ml}$ de méthylate de sodium, $600 \mathrm{ml}$ de méthanol et un peu de pierre ponce, est adapté un réfrigérant à reflux. Le ballon est placé dans un bainmarie et porté à ébullition sous azote pendant 2 à 3 heures jusqu'à obtention d'une seule phase. Après refroidissement à température ambiante (sous azote), le mélange se sépare en deux phases :

- la phase supérieure est constituée par les esters méthyliques dans le méthanol,

- la phase inférieure est constituée par le glycérol.

L'excès de sodium qui se trouve dans la phase des esters méthyliques est neutralisé par l'acide chlorhydrique (vérification au papier $\mathrm{pH}$ ).

La solution est versée dans une ampoule à décanter et les esters sont insolubilisés partiellement en ajoutant $100 \mathrm{ml}$ d'eau distillée. Après agitation et repos, le mélange se sépare en deux phases :

- la phase inférieure est constituée par les esters méthyliques purs,

- la phase supérieure est constituée par le mélange méthanol-eau. Une partie des esters méthyliques y reste en solution.

Après avoir soutiré et mis de côté la phase inférieure, les esters restant dans la phase supérieure sont extraits par l'hexane.

La solution hexanique est ajoutée aux esters mis de côté, le solvant est distillé sous vide et les esters sont pesés.

TABI,EAU I

Composition en acides gras des fractions de cristallisation de l'huile d'olive (en p. roo des esters méthyliques)

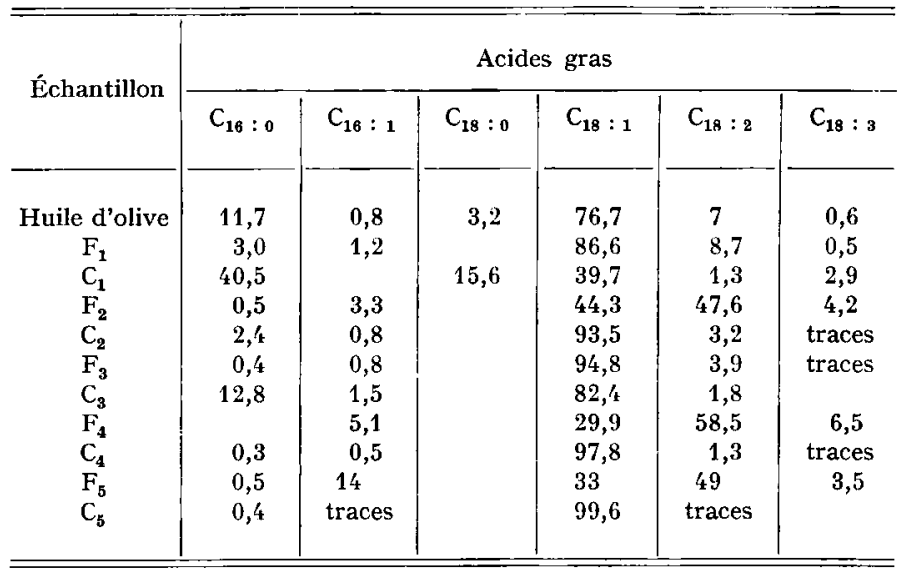

\section{Cristallisation à l'urée.}

Les différentes phases de cristallisation sont résumées dans la figure r. Aux esters méthyliques des acides gras sont ajoutés un poids égal d'urée et un volume to fois supérieur de méthanol. La solubilisation s'effectue par chauffage au bain-marie.

La solution est placée dans une chambre à $4^{\circ} \mathrm{C}$ pendant une nuit et filtrée sur verre fritté à la même température. Les cristaux sont essorés sous vide. Au filtrat $F_{1}$ est ajoutée une quantité 
d'urée égale à đeux fois la quantité introduite au début. Après dissolution, le mélange est placé à $4^{\circ} \mathrm{C}$, les cristaux $\mathrm{C}_{2}$ formés sont filtrés, rincés avec du méthanol à $4^{\circ} \mathrm{C}$ et remis en solution dans un volume de méthanol égal à $\mathbf{I}, 5$ fois le volume utilisé pour la première cristallisation. La cristallisation se fait alors à la température iambiante.

$\mathrm{Au}$ filtrat $\mathrm{F}_{3}$ est ajoutée $\mathrm{I}, 2$ fois la quantité d'urée utilisée à la première cristallisation. Après formation des composés d'inclusion à $4^{\circ} \mathrm{C}$, les cristaux $\mathrm{C}_{4}$ sont conservés. L'urée est séparée des esters méthyliques par un lavage avec une solution chaude d'acide chlorhydrique à I p. Ioo. L'urée soluble dans la phase eau-acide est éliminée par décantation. Les esters méthyliques repris par l'hexane sont lavés à l'eau pure pour enlever l'excès d'acide, puis séchés sur sulfate de sodium. Le solvant est évaporé sous vide et les esters $\mathrm{C}_{4}$ sont pesés.

La dernière cristallisation des esters méthyliques est effectuée à $-60^{\circ} \mathrm{C}$ en plaçant une fiole contenant la solution à Io $\mathrm{p}$. Ioo des esters dans l'acétone à l'intérieur d'un bac contenant de la neige carbonique. Après 3 ou 4 heures, l'acide oléique ayant cristallisé, le mélange est filtré sur verre fritté refroidi. Après distillation du solvant restant, il reste les esters $\mathrm{C}_{5}$.

Au cours des différentes opérations, les fractions obtenues sont analysées par chromatographie gaz-liquide.

Les conditions opératoires sont les suivantes :

- Appareil Microtek GC $2500 \mathrm{R}$

longueur de la colonne : $3 \mathrm{~m}$, diamètre $\mathrm{r} / 8 \mathrm{in}$.

phase stationnaire : polymère EG SSX à la concentration de $5 \mathrm{p}$. Ioo sur du chromosorb G DMCS 80/100 mesh

gaz vecteur : azote

$t$ de la colonne $\mathrm{I} 70^{\circ} \mathrm{C}$

nombre de plateaux théoriques: environ 2200

Détecteur à ionisation de flamme.

Intégrateur électromécanique.

Une colonne capillaire ayant un grand pouvoir séparateur permet la détection des isomères de l'acide oléique obtenu à la dernière cristallisation.

- Appareil Girdel 3 ooo

longueur de la colonne : Ioo $\mathrm{m}$, diamètre $0,5 \mathrm{~mm}$

phase stationnaire : BDS

gaz vecteur : azote

$i$ de la colonne : $175^{\circ} \mathrm{C}$

nombre de plateaux théoriques: environ 62000 .

- Détecteur à ionisation de flamme.

- Intégrateur électronique.

Le tableau I donne la composition des fractions obtenues au cours de l'une des préparations et la figure 2 l'enrichissement des fractions en oléate de méthyle.

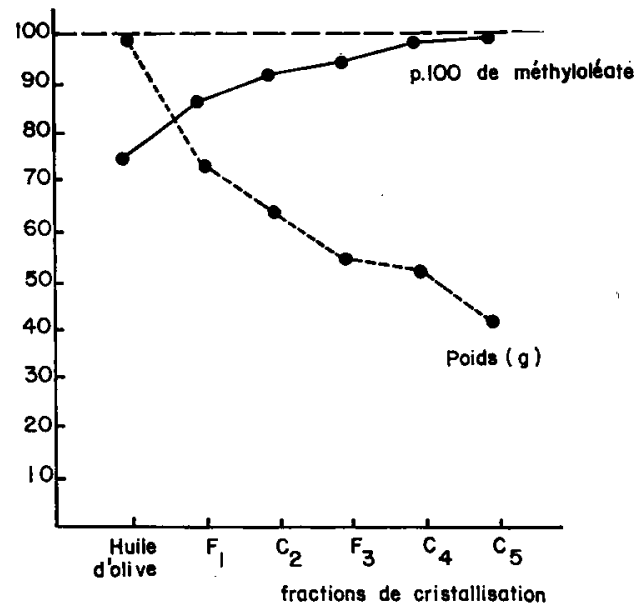

FIG. 2. - Enrichissement en oléate de méthyle

et rendement pondéral aux différentes étapes de la cristallisation 
La pureté de l'oléate de méthyle est dans ce cas de 99,6 p. roo.

Pour isoler l'acide oléique, il suffit de saponifier les esters méthyliques et de libérer les acides gras par l'acide chlorhydrique.

L'acide oléique existe sous deux formes, l'une fondant à $13^{\circ} \mathrm{C}$ et l'autre à $\mathrm{I} 6^{\circ} \mathrm{C}$. Le point de fusion déterminé au laboratoire avec un tube de Thiele se situe entre $14^{\circ} \mathrm{C}$ et $15^{\circ} \mathrm{C}$ selon les échantillons. Le point de solidification est compris entre $8^{\circ} \mathrm{C}$ et $9^{\circ} \mathrm{C}$.

\title{
DISCUSSION
}

La cristallisation avec l'urée est effectuée à partir des esters méthyliques, mais elle pourrait l'être également à partir des acides gras.

Le choix des esters méthyliques présente l'avantage de permettre à tout moment l'analyse par chromatographie gaz-liquide des fractions obtenues lors des cristallisations. En outre, nous avons constaté qu'avec les acides gras la pureté de l'acide oléique est inférieure, car l'élimination des acides saturés est moins poussée qu'avec les esters méthyliques.

D'une façon générale, il est préférable d'effectuer la méthylation sur les acides gras obtenus après saponification de l'huile plutôt que directement sur cette dernière, de façon à pouvoir éliminer l'insaponifiable. Avec la technique de cristallisation à l'urée, l'insaponifiable ne formant pas d'inclusions se trouve rejeté lors des filtrations.

Le rendement de la préparation est satisfaisant, puisque environ $56 \mathrm{p}$. roo de l'acide oléique contenu dans l'huile d'olive sont récupérés (43 p. roo du poids des esters méthyliques des acides gras de l'huile d'olive, dont la teneur en oléate de méthyle était de 76,7 p. Ioo).

La pureté de l'acide oléique est supérieure à $99,5 \mathrm{p}$. Ioo. La proportion de l'acide palmitique restant varie légèrement d'une préparation à l'autre autour de $0,4 \mathrm{p}$. Ioo. L'analyse sur colonne capillaire montre qu'il reste des traces d'isomères $\omega 7$ de l'acide oléique. Elle met en évidence la présence de traces d'acide linoléique et d'acides monoinsaturés $\left(C_{16: 1}, C_{12: 1}, C_{20: 1}\right)$. Ces derniers, bien que présents en très faible quantité dans l'huile d'olive, ne sont pas totalement éliminés au cours des cristallisations, car leurs solubilités sont très voisines de celles de 1'acide oléique. L'isolement intégral de cet acide gras peut être obtenu par une chromatographie gaz-liquide préparative.

Reçu pour publication en mai 1973.

\section{SUMMARY}

\author{
PREPARATION OF OLEIC ACID AT 99. 5. IOO PURITY
}

Oleic acid is isolated from methyl esters of olive oil fatty acids. Using the technique of inclusion with urea, saturated fatty acids and most of the unsaturated fatty acids are eliminated. After four crystallizations at $+4^{\circ} \mathrm{C}$ or at $+20^{\circ} \mathrm{C}$, less than 5 p. roo of other methyl esters remain with the methyl oleate. A last ester crystallization is done in acetone at $-60^{\circ} \mathrm{C}$. Oleic acid having a degree of purity equal to or higher than $99.5 \mathrm{p}$. Ioo is obtained with an ester hydrolysis.

\section{RÉFÉRENCES BIBL,IOGRAPHIQUES}

Brown J.-B., r955. Fractional solvent crystallization. J. Am. Oil Chem. Soc., 32, 646-652.

Brown J.-B., KolB D. K., 1955. Applications of low-temperature crystallization in the separation of the fatty acids and their compounds. In Progress in the chemistry of fats and other lipids, 3, 58-94. Pergamon Press Ltd, London. 
Fedeli E., Camurati F., Lanzani A., I969. Preparazione di acidi grassi puri. Riv. Ital. Sost. Grasse, 46, 5I4-5I9.

Kepler J. G., Sparreboom S., Stroink J. B. A., Von Mikusch J. D., i959. A note on the preparation of pure oleic and linoleic acid. J. Am. Oil Chem. Soc., 36, 308-309.

Loury M., I968. Les procédés physiques de séparation. Séparation par changements d'état : distillation, entraînement, cristallisation, inclusion. Journées Inform. 1968, Méth. instrum. Anal. Contrôle Corps gras Prod. app., Paris, 27-31 mai, 59-70.

MurRay K. E., r955. Low-pressure fractional distillation and its use in the investigation of lipids. In Progress in the chemistry of fats and other lipids, 3, 244-273. Pergamon Press Ltd, London.

QuinN J. G., SAmpugna J., Jensen R. G., I967. Synthesis of Ioo-gram quantities of highly purified mixed acid triglycerides. J. Am. Oil Chem. Soc., 44, 440-442.

Rubin L. J., Paisley W., ig6o. Pure oleic acid from olive oil. J. Am. Oil Chem. Soc., 37, 300-302.

Schlenk H., I954. Urea inclusion compounds of fatty acids. In Progress in the chemistry of fats and other lipids, 2, 243-267. Pergamon Press Ltd, London.

Schlenk H., rg6r. Crystallization of fatty acids. J. Am. Oil. Chem Soc, 38, 728-736.

Schlenk H., Holman R. T., r950. Separation and stabilization of fatty acids by urea complexes. J. Am. Oil Chem. Soc., 72, 5001-5004.

Sweris D., I964. E. Urea complexes. 6. Purification of derived compounds : a) Oleic acid and methyloleate. In MARKLEY K. S. Fatty acids. Their chemistry, properties, production and uses, 3, 2327-2329. Interscience publishers, New York, London, Sydney.

SWERN D., PARker W. E., 1952. Application of urea complexes in the purification of fatty acids, esters, and alcohols. II. Oleic acid and methyl oleate from olive oil. J. Am. Oil Chem. Soc., 29, 614-615, 